\title{
Extreme Frequency Conversion from Soliton Resonant Interactions
}

\author{
Myungwon Hwang and Andres F. Arrieta* \\ School of Mechanical Engineering, Purdue University, West Lafayette, Indiana 47907, USA
}

(Received 14 June 2020; accepted 27 January 2021; published 19 February 2021)

\begin{abstract}
We present a metastructure architecture with a bistable microstructure that enables extreme broadband frequency conversion. We use numerical and experimental tools to unveil the relationship between input excitations at the unit cell level and output responses at the macrostructural level. We identify soliton-lattice mode resonances resulting in input-independent energy transfer into desired metabeam vibration modes as long as transition waves are triggered within the metastructure. We observe both low-to-high and high-tolow incommensurate frequency interactions in the metabeams, thus enabling energy exchange between bands 2 orders of magnitude apart. This behavior generalizes fluxon-cavity mode resonance in superconducting electronics, providing a general method to extreme frequency conversion in mechanics. Importantly, the introduced architecture allows for expanding the metamaterials design paradigm by fundamentally breaking the dependence of macroscopic dynamics on the unit cell properties. The resulting input-independent nature implies potential applications in broadband frequency regulation and energy transduction.
\end{abstract}

DOI: 10.1103/PhysRevLett.126.073902

Introduction.-Solitary waves appear in various physical systems [1] playing a pivotal role in applications, including waveguiding [2], photonics [3], optical communications [4], reversible logic gates [5], lasing [6], morphing structures [7], nondestructive testing [8], and soft robotics [9]. A unique aspect of solitons is their quasiparticle characteristics. This allows for better imaging using sonic bullets [10] or dense wavelength-division multiplexing for optical communications exploiting cavity solitons [11]. Solitons' resonant interactions with cavities have enabled the generation of lasers exhibiting frequency conversion not readily available with conventional sources exploiting fluxons-a type of transition wave or topological soliton -in Josephson junctions [12]. Such extreme frequency conversion in mechanical systems is less common and is typically achieved via subharmonic, superharmonic, or combination resonances [13] and nonlinear coupling between normal modes [14]. Nonlinear multimode metamaterials can provide alternate ways to attain frequency conversion through their amplitude-dependent dispersion relations $[15,16]$. Intermodal interactions through nonlinearly generated higher harmonics have been theoretically and experimentally demonstrated, enabling energy exchange between different wave modes [17-19]. Furthermore, magnetic metamaterials featuring engineered

Published by the American Physical Society under the terms of the Creative Commons Attribution 4.0 International license. Further distribution of this work must maintain attribution to the author(s) and the published article's title, journal citation, and DOI. defects have demonstrated conversion from high- to lowfrequency modes through resonant coupling between localized defect modes and extended lattice modes [20]. However, to excite these interaction mechanisms, the defect mode's frequency needs to be commensurate with or a combination of the system's modes, still limiting the attainable frequency bandwidth.

Lattices composed of bistable elements can support transition waves exhibiting particlelike behavior [21-23]. When the constitutive on-site members are built with asymmetric bistable elements, transition waves become unidirectional $[24,25]$ and exhibit unique input-independent dynamics [26], implying strong potential for broadband applications. Enabling nonlinear interactions similar to fluxon-cavity mode resonances exploiting the input frequency independence of transition waves offers the potential for extreme energy conversion currently absent in mechanical systems. In this study, we demonstrate extreme energy exchange in mechanical systems with metabeams composed of a bistable microstructure that promotes nonlinear coupling between wave and metastructural modes in an analogous process to fluxons interacting with cavity modes in superconductors $[6,12]$. Importantly, the soliton resonant interactions enable extreme frequency conversion and response manipulation in mechanical systems, without recourse to dispersion-related phenomena. The unveiled dynamics are insensitive to the excitation frequency, thus laying the foundation for a new physical mechanism, the dynamics of which are independent of the metamaterial building block size.

Experimental results.-To illustrate the extreme frequency conversion phenomenon, we manufacture and test 
a metabeam featuring an engineered bistable microstructure. The model metabeam is built by $3 \mathrm{D}$ printing a ladderlike structure [black component in Fig. 1(a)], the rungs of which are interconnected by springlike features providing intersite interaction. The intersite members are aligned at a small offset distance from the metabeam's center line. This design feature is introduced to break the cross section's symmetry about the center line, allowing the excitation of the bending modes by in-plane inputs (see Movie S1 [27]). The metabeam is then fitted between a set of flexible holders [white components in Fig. 1(a)], providing flexibility in the transverse direction and allowing the microstructure to exhibit two different stable states [red and green dashed lines in Figs. 1(a)(i) and 1(a)(ii)]. In this example setup, one end of the structure is clamped to realize a fixed-free boundary condition. A harmonic displacement input is imposed at the first element in the in-plane direction through an electrodynamic shaker. The input and output displacements are measured by a set of laser displacement sensors, pointing at the flat surfaces of the shaker head and near the free end of the structure, respectively. The overall experimental setup is shown in Fig. 1(a)(iii), and more detailed fabrication and experimental methods are given in Sec. 1 of Supplemental Material [27].

To summarize the input and output frequency relations, we construct an output frequency diagram [Figs. 1(b) and 1(c)]. The diagram summarizes up to the 20 largest peaks of the output displacement's power spectral density (DPSD)
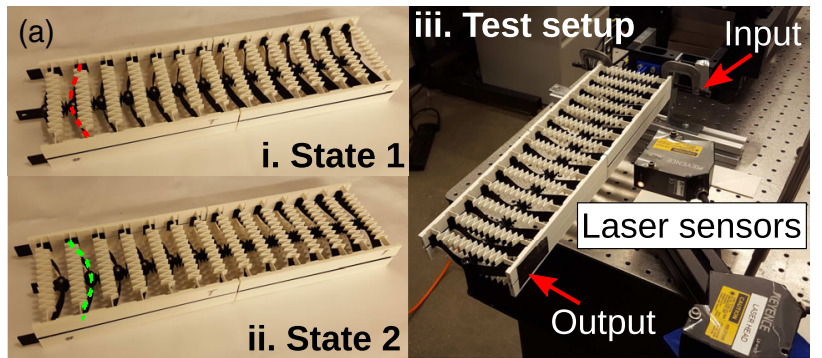

(b) Small amplitude

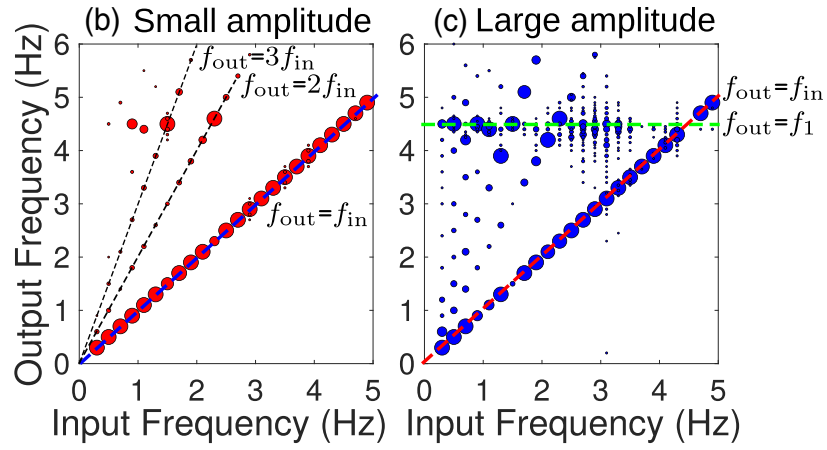

FIG. 1. (a) Experimental demonstrator and shaker test setup. Experimental output frequency diagrams under (b) small- and (c) large-amplitude excitations. $f_{\text {out }}, f_{\text {in }}$, and $f_{1}$ are the output frequency, input frequency, and fundamental mode of the metabeam, respectively. as a function of the input frequency. Each dot in the diagram represents the available frequency component of the output response, while its size indicates the relative intensity: A larger dot indicates larger spectral contribution. For each input cases, the relative intensity $r_{\mathrm{DPSD}}$ is calculated by DPSD $i /$ DPSD $_{\text {max }}$, where $\mathrm{DPSD}_{i}$ is the power of the $i$ th frequency component and DPSD $\mathrm{max}_{\text {ax }}$ is the power of the most dominant peak. DPSD $_{i}$ 's less than $0.1 \%$ of DPSD $_{\max }$ are discarded to declutter the output frequency diagrams. For better visualization of the subdominant frequency peaks, the dots are logarithmically scaled such that they appear as $2^{\log _{10} r_{\mathrm{DPSD}}}$ in size. Blue dots indicate the generation of in-plane transition waves within the metabeam, while red dots represent responses involving no phase transitions. The DPSD is obtained when the system attains steady state with the aid of welch function in PYTHON's scipy library at $1000 \mathrm{~Hz}$ sampling frequency without windowing.

For a relatively small input displacement about one of the stable states for which transition waves are not triggered, the dominant output frequency corresponds to either the input frequency or its harmonics close to the fundamental mode (4.62 Hz [27]) of the metabeam [Fig. 1(b)]. The harmonic contributions' appearance is due to the experimental limitation in generating purely sinusoidal displacement signals with our test setup, leading excitation of superharmonics of the input frequency (Sec. 2 of Supplemental Material [27]). In this low forcing amplitude regime, the observed dynamics are fully accountable in terms of the typical linear and weakly nonlinear responses that do not involve the triggering of transition waves.

Increasing the imposed displacement amplitude further triggers transition waves, revealing an output frequency branch close to the structural mode [green dashed line in Fig. 1(c) and Movie S2] in addition to a branch corresponding to the input frequencies (red dashed line). Interestingly for the input frequencies above $2.5 \mathrm{~Hz}$, the frequencies of this subdominant output branch are not the results of superharmonic resonance [see the spectral content around $4.5 \mathrm{~Hz}$ in Figs. S2(e) and S2(f)]; rather, these exhibit incommensurate relations with the input frequencies. Our experiments thus reveal that the generation of transition waves results in a solution branch showing coherent output frequency around the fundamental mode $f_{1}$ of the metabeam unrelated to the input frequencies. Notably, the transition wave generation yields a fundamental mechanism to achieve incommensurate frequency resonance displaying strong stability, which is uncommon in structural systems [30]. As this dynamics manifests when transition waves (i.e., topological solitons) are triggered, we referred to this phenomenon as solitonic resonance.

The solitonic resonance can be explained by the transition wave's particlelike nature [1,31]. In this regime, the generated quasiparticles impulsively excite the metastructure continuously. This triggers lasting transients even 


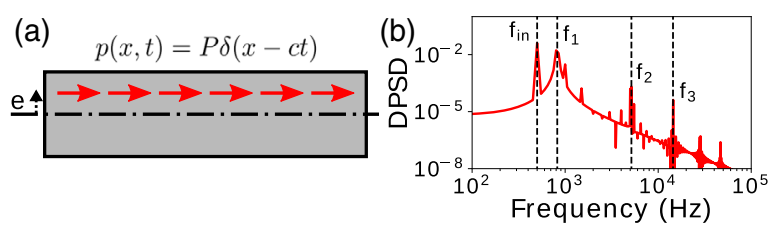

FIG. 2. (a) Schematic representation of a beam subjected to a moving particle. (b) Power spectral density of the approximated tip displacement of the beam in a fixed-free configuration.

in a nonconservative system. This mechanism is similar to frequency up-conversion in devices [32], but our metabeam utilizes the quasiparticle motion instead of direct impacts. We can further analogize the metabeam's solitonic resonance employing an Euler-Bernoulli beam subjected to an in-plane traveling particle. The motion of a single particle moving along the beam's length can be mathematically represented as a localized traveling impulse [Fig. 2(a)]. This beam problem can be solved exactly by a modal expansion method (see Sec. 4 of Supplemental Material [27] for the analysis details). An example five-term approximation of the tip displacement for such a beam in a fixed-free configuration is obtained by sending 22 particles every $2 \mathrm{~ms}$. The resulting DPSD obtained for the response between 24 and 44 ms [Fig. 2(b)] confirms that a moving impulse indeed excites the normal modes $f_{n}$ 's of the beams.

Numerical results.-Although the postbuckled beam design as the microstructure readily yields bistability in an experimental demonstrator, a spring-joined bistable unit cell design is more advantageous for a simpler mathematical description. Thus, we adopt it in the subsequent numerical investigation by constructing a beamlike frame with underlying spring-mass bistable microstructures [23]. We introduce an offset from the center line of the frame [dash-dotted line in Fig. 3(a)] to break the system's crosssectional symmetry and amplify the flexural motion. The buckled beam, providing the local bistability, is idealized by a pair of linear springs with the constants $k_{2}$ and $k_{3}$. The spring with the constant $k_{1}$ serves as the intersite connection between neighboring on-site members. The flanges and the holders of the experimental demonstrator form an external elastic frame, which is idealized by the rest of the linear springs $\left(k_{4}-k_{8}\right)$ in the mathematical design. Additionally, small mass proportional damping is imposed across the metabeam to suppress any unwanted transient effects.

A fixed-free boundary condition is used; however, the ensuing dynamics are independent of the boundary conditions (Sec. 6 of Supplemental Material [27]). Harmonic displacement input $u_{1}(t)=R-A \cos \left(2 \pi f_{\text {in }} t-\phi_{0}\right)$ or force input $p(t)=F \sin 2 \pi f_{\text {in }} t$ are used to separately excite the metabeam [33]. The excitation is applied in the in-plane direction at the leftmost node of the internal bistable lattice, where $A, \phi_{0}$, and $F$ are the displacement amplitude, phase constant, and forcing amplitude, respectively. The output displacement is measured at the top rightmost node in the out-of-plane direction. See Fig. 3(a) and Sec. 5 of Supplemental Material [27] for the full metabeam description and design parameters. The metabeam's dynamics are simulated using ABAQUS/STANDARD implicit nonlinear solver and reported in output frequency diagrams.

We first construct a numerical model qualitatively similar to the experimental metabeam, which is achieved by matching the unit cell natural frequency and the fundamental macrostructural mode of the numerical model to those of the experimental metabeam (see Supplemental Material [27] for the design parameters). The simulated output response diagrams display qualitative agreement with the experimental response. The metabeam under $1 \mathrm{~mm}$ input amplitude shows purely linear behavior, confirming that the solitonic resonance is not a product of the peculiar unit cell design [Fig. 3(b)]. Under $9.5 \mathrm{~mm}$ input amplitude, transition waves are triggered within the metabeam, resulting in coexisting linear and solitonic resonance solution branches [red and green dashed lines in Fig. 3(c)]. The numerical simulations for this case are then extended to a higher input frequency range, still showing the output frequency branch concentrated at $\sim 4.62 \mathrm{~Hz}$ [34] but almost eliminating the branch corresponding to the input frequencies. This disappearance of the input frequency contribution is due to the aperiodic generation of the transition waves. At low input frequencies, such that the snap-through transitions at the excitation site occur at a much slower rate than the transition wave's propagation speed, each unit's interwell oscillation takes place at the same frequency as the input frequency, resulting in the direct contribution from the input. In contrast, increasing the input frequency

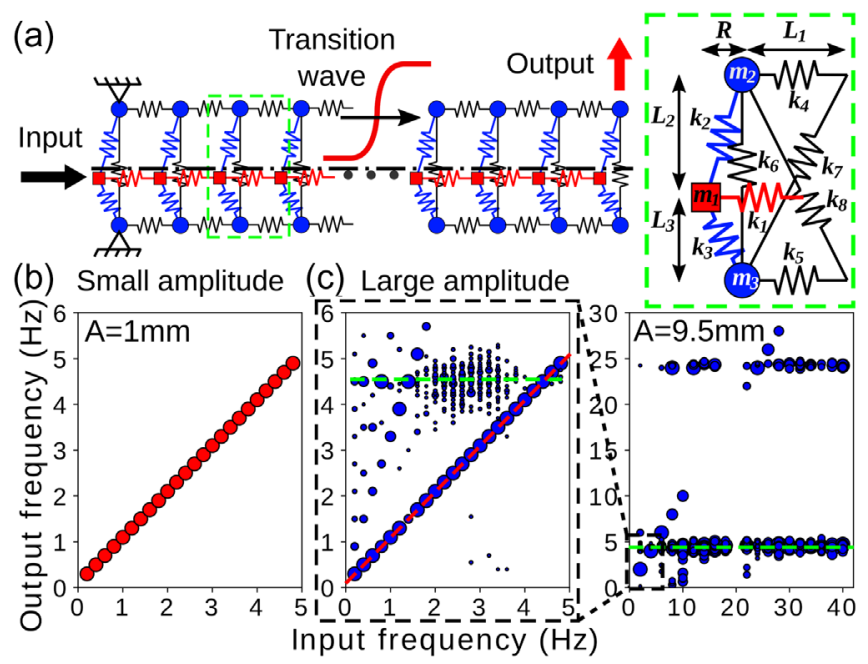

FIG. 3. (a) Schematic representation of the metabeam. Output frequency diagrams for a qualitatively similar numerical design under (b) small- and (c) large-amplitude excitations. The enlarged region shows the low-frequency responses corresponding to the experimental range. 
results in a faster transition wave generation rate so that the newly generated and previously traveling transition waves interact with one another. Thereby, the resulting interwell oscillations occur at nondefinite rates, imposing the prevalence of transient effects in the response. The transient effect's dominance over the input frequency effect can be further manipulated by the system damping as long as manufacturing technology allows (Sec. 7 of Supplemental Material [27]).

Even more extreme energy transfer can be achieved, employing the force input, since the induced snap-through instability is chaotic in nature [35], hence facilitating the aperiodic generation of transition waves. As a baseline design, the spring constants are tuned to have the fundamental macroscopic mode at $26.3 \mathrm{~Hz}$ and the unit cell mode at $39.7 \mathrm{~Hz}$ (see Supplemental Material [27] for the design parameters). Transition waves can be excited via quasistatic or dynamic inputs but are more easily triggered by harmonic excitations around the unit cell mode, as shown in the response map [Fig. 4(a)]. The map indicates whether transition waves are generated for the given input amplitude and frequency combinations. Furthermore, the frequency range expands with increasing forcing amplitudes.

The particlelike nature of the transition waves can be clearly identified by the stable propagation of the kinkshaped waveform in Fig. 4(b)(i), showing the in-plane displacement $u_{1}$ of the center mass in the space configuration under a quasistatic $(1 \mathrm{~Hz})$ input. For higher input frequencies [36 Hz in Fig. 4(b)(ii)], the waveform is distorted by nonlinear interactions with other lattice modes. Nevertheless, the waveform starting at one stable state and ending at another is readily identifiable. In addition, the induced transition waves provoke response amplification as in mechanical resonance. Figure 4(c) shows the responses just below and above the forcing amplitude triggering transition waves. With only $3.4 \%$ increase in the forcing amplitude, the output displacement $w_{2}$ amplifies as much as 14 times (Sec. 8 of Supplemental Material [27]).

Figure 4(d)(i) shows the output frequency diagram for the baseline design under $F=1 \mathrm{~N}$, and one representative response $\left(f_{\text {in }}=36 \mathrm{~Hz}\right)$ when transition waves are generated is given in Fig. 4(e). The resulting power spectral densities of $w_{2}$ 's again confirm that the generation of transition waves excites the macroscopic metastructure's fundamental mode [dashed line in Fig. 4(d)(i)]. Compared to the displacement input cases, however, almost complete energy transfer into this macroscopic mode can be achieved due to the aperiodic generation of transition waves, illustrated by the nondefinite state transition rates of the in-plane displacement $u_{1}$ at the excitation site [Fig. 4(e)(i)]. The effect of the generation rate disperses in the frequency spectrum, leaving the transient effect to dominate the outof-plane response. As the forcing amplitude increases to $1.7 \mathrm{~N}$, the input bandwidth yielding solitonic resonance broadens [Fig. 4(d)(ii) and Movie S3]. It is further observed that even noisy input sources can be transformed into a single coherent response mode (Sec. 9 of Supplemental Material [27]). Thus, the strongly nonlinear dynamics displayed by the proposed metastructures enable a robust mechanism for broadband frequency conversion into a coherent output frequency independently from the excitation frequencies.

Other potentially useful nonlinear behaviors exist outside the solitonic resonance domain, such as second
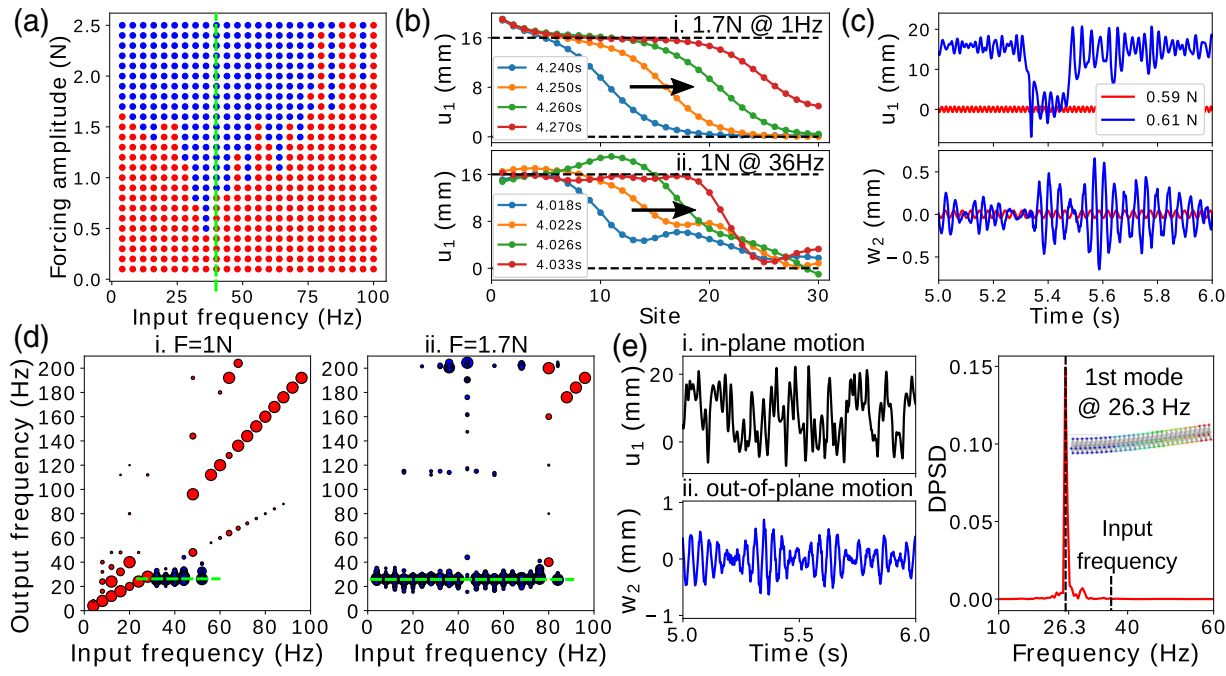

FIG. 4. (a) Response map in terms of input forcing amplitude and frequency, where blue dots indicate that transition waves are triggered. (b) In-plane displacements of the center masses in space configuration, showing transition wave propagation. (c) Response amplification when transition waves are triggered (in blue) compared to when they are not triggered (in red). (d) Output frequency diagrams for various input conditions, showing the output frequency spectrum for each input frequency. (e) Representative response of solitonic resonance, obtained for $F=1 \mathrm{~N}$ at $36 \mathrm{~Hz}$. 
(a) Design B-1:

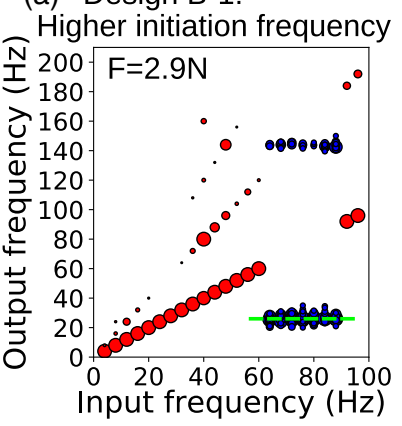

(c) Design C: Low-to-high frequency conversion

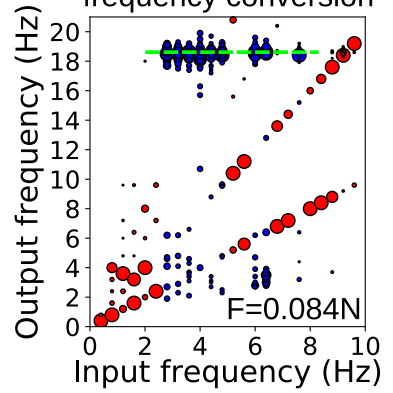

(b) Design B-2:

Higher output frequency

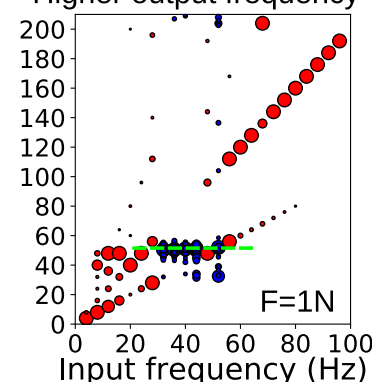

(d) Design D: High-to-low 1000 frequency conversion

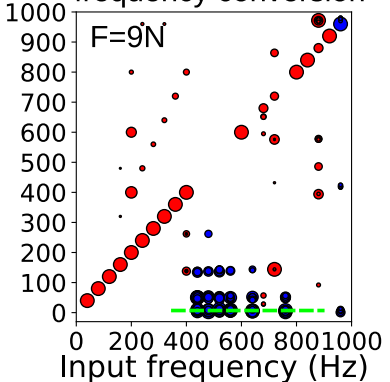

FIG. 5. Output frequency diagrams for various unit cell and external frame design combinations, demonstrating extreme tuning flexibility. (a) Design B-1 increases the initiation frequency of the solitonic resonance, and (b) design B-2 increases the output frequency. Output frequency diagrams for metabeam designs tuned to enable (c) low-to-high and (d) high-to-low frequency conversions, where the input and output frequencies are 1-2 orders of magnitude apart.

harmonic generation [between 12 and $20 \mathrm{~Hz}$ in Fig. 4(d)(i)] or frequency doubling (above $56 \mathrm{~Hz}$ ) (Sec. 10 of Supplemental Material [27]). These will be addressed in detail in a separate study.

Tuning flexibility.-The simplicity and periodicity of the proposed metastructure allow the operating bandwidth to be easily tuned to suit a vast range of applications. The unit cell design dictates the central input frequency around which the solitonic resonance emerges, while the overall metastructure topology controls the corresponding output frequency. Quadrupling $k_{2}$ and $k_{3}$ doubles the in-plane unit cell mode, thus shifting up the input frequency inducing solitonic resonance [Fig. 5(a)]. On the other hand, quadrupling $k_{4}-k_{8}$ approximately doubles the macrostructure's original natural frequencies, moving the output frequency of solitonic resonance to a higher frequency range [Fig. 5(b)].

The metabeam can also be tuned to display remarkable energy exchanges between incommensurate frequency bands, which can be separated by several orders of magnitude. Two such examples are presented (see Supplemental Material [27] for the design parameters): one optimized to operate at $3-5 \mathrm{~Hz}$ range yielding $\sim 18.63 \mathrm{~Hz}$ solitonic resonance [Fig. 5(c)] and the other

at $400-560 \mathrm{~Hz}$ range yielding solitonic resonance below $10 \mathrm{~Hz}$ [Fig. 5(d)]. The tuning flexibility in the presented metastructures thus yields a blueprint for unprecedented dynamical manipulation from an intrinsically nonlinear interaction.

Conclusions.-We demonstrate nonlinear interactions involving solitary wave and vibration modes, resulting in extreme frequency conversion in generic bistable metastructures. Notably, the observed phenomenon referred to as solitonic resonance allows for a remarkably stable means to achieve input-independent frequency conversion, in which the output response is unaffected by wave dispersion. This behavior generalizes analogous fluxoncavity mode resonance in superconducting electronics, providing an exemplary platform for frequency regulation and energy conversion applications across physical fields. The tuning flexibility of the presented metabeam also allows for extreme energy exchange between incommensurate frequencies separated by orders of magnitude. Importantly, the presented architecture provides a general model system for realizing unique properties in metamaterials at frequencies not dictated by the unit cell, opening up new avenues for attaining extreme dynamics.

The authors thank Professor C. Scalo for supporting the development of the in-house code. The authors are grateful for Dr. E. Arrieta Diaz's help in motivating this work beyond mechanics. We acknowledge the financial support from the National Science Foundation under Grant No. CMMI-1935137.

*aarrieta@purdue.edu

[1] M. Remoissenet, Waves Called Solitons: Concepts and Experiments (Springer, Berlin, 1996).

[2] D. N. Christodoulides and E. D. Eugenieva, Blocking and Routing Discrete Solitons in Two-Dimensional Networks of Nonlinear Waveguide Arrays, Phys. Rev. Lett. 87, 233901 (2001).

[3] D. N. Christodoulides, F. Lederer, and Y. Silberberg, Discretizing light behaviour in linear and nonlinear waveguide lattices, Nature (London) 424, 817 (2003).

[4] P. Marin-Palomo, J. N. Kemal, M. Karpov, A. Kordts, J. Pfeifle, M. H. P. Pfeiffer, P. Trocha, S. Wolf, V. Brasch, M. H. Anderson, R. Rosenberger, K. Vijayan, W. Freude, T. J. Kippenberg, and C. Koos, Microresonator-based solitons for massively parallel coherent optical communications, Nature (London) 546, 274 (2017).

[5] W. Wustmann and K. D. Osborn, Reversible fluxon logic: Topological particles allow ballistic gates along onedimensional paths, Phys. Rev. B 101, 014516 (2020).

[6] M. C. Cassidy, A. Bruno, S. Rubbert, M. Irfan, J. Kammhuber, R. N. Schouten, A. R. Akhmerov, and L. P. Kouwenhoven, Demonstration of an ac Josephson junction laser, Science $\mathbf{3 5 5}$, 939 (2017).

[7] A. Zareei, B. Deng, and K. Bertoldi, Harnessing transition waves to realize deployable structures, Proc. Natl. Acad. Sci. U.S.A. 117, 4015 (2020). 
[8] T. Singhal, E. Kim, T.-Y. Kim, and J. Yang, Weak bond detection in composites using highly nonlinear solitary waves, Smart Mater. Struct. 26, 055011 (2017).

[9] B. Deng, L. Chen, D. Wei, V. Tournat, and K. Bertoldi, Pulse-driven robot: Motion via solitary waves, Sci. Adv. 6, eaaz1166 (2020).

[10] A. Spadoni and C. Daraio, Generation and control of sound bullets with a nonlinear acoustic lens, Proc. Natl. Acad. Sci. U.S.A. 107, 7230 (2010).

[11] A. M. Weiner, Cavity solitons come of age, Nat. Photonics 11, 533 (2017).

[12] L. Ozyuzer, A. E. Koshelev, C. Kurter, N. Gopalsami, Q. Li, M. Tachiki, K. Kadowaki, T. Yamamoto, H. Minami, H. Yamaguchi, T. Tachiki, K. E. Gray, W. K. Kwok, and U. Welp, Emission of coherent $\mathrm{THz}$ radiation from superconductors, Science 318, 1291 (2007).

[13] A. H. Nayfeh and D. T. Mook, Nonlinear Oscillations (Wiley, New York, 1995).

[14] P. Malatkar and A. H. Nayfeh, On the transfer of energy between widely spaced modes in structures, Nonlinear Dyn. 31, 225 (2003).

[15] R. K. Narisetti, M. J. Leamy, and M. Ruzzene, A perturbation approach for predicting wave propagation in onedimensional nonlinear periodic structures, J. Vib. Acoust. Trans. ASME 132, 0310011 (2010).

[16] R. Khajehtourian and M. I. Hussein, Dispersion characteristics of a nonlinear elastic metamaterial, AIP Adv. 4, 124308 (2014).

[17] R. Ganesh and S. Gonella, Nonlinear waves in lattice materials: Adaptively augmented directivity and functionality enhancement by modal mixing, J. Mech. Phys. Solids 99, 272 (2017).

[18] R. Ganesh and S. Gonella, Experimental evidence of directivity-enhancing mechanisms in nonlinear lattices, Appl. Phys. Lett. 110, 084101 (2017).

[19] X. Fang, J. Wen, D. Yu, G. Huang, and J. Yin, Wave propagation in a nonlinear acoustic metamaterial beam considering third harmonic generation, New J. Phys. 20, 123028 (2018).

[20] M. Serra-Garcia, M. Molerón, and C. Daraio, Tunable, synchronized frequency down-conversion in magnetic lattices with defects, Phil. Trans. R. Soc. A 376, 20170137 (2018).

[21] L. I. Manevich, A. V. Savin, V. V. Smirnov, and S. Volkov, Solitons in nondegenerate bistable systems, Usp. Fiz. Nauk 164, 937 (1994).

[22] A. Cherkaev, E. Cherkaev, and L. Slepyan, Transition waves in bistable structures. I. Delocalization of damage, J. Mech. Phys. Solids 53, 383 (2005).
[23] N. Nadkarni, C. Daraio, and D. M. Kochmann, Dynamics of periodic mechanical structures containing bistable elastic elements: From elastic to solitary wave propagation, Phys. Rev. E 90, 023204 (2014).

[24] N. Nadkarni, A. F. Arrieta, C. Chong, D. M. Kochmann, and C. Daraio, Unidirectional Transition Waves in Bistable Lattices, Phys. Rev. Lett. 116, 244501 (2016).

[25] J. R. Raney, N. Nadkarni, C. Daraio, D. M. Kochmann, J. A. Lewis, and K. Bertoldi, Stable propagation of mechanical signals in soft media using stored elastic energy, Proc. Natl. Acad. Sci. U.S.A. 113, 9722 (2016).

[26] M. Hwang and A. F. Arrieta, Input-independent energy harvesting in bistable lattices from transition waves, Sci. Rep. 8, 3630 (2018).

[27] See Supplemental Material at http://link.aps.org/ supplemental/10.1103/PhysRevLett.126.073902 for additional details about the metabeam designs, experimental method, theoretical investigation, and numerical results, which include Ref. [28,29]. Movie S1 illustrates the coupling between the in-plane and out-of-plane motions. Movie S2 and S3 show the experimental and numerical demonstrations of solitonic resonance.

[28] H. M. Hilber, T. J. R. Hughes, and R. L. Taylor, Improved numerical dissipation for time integration algorithms in structural dynamics, Earthquake Eng. Struct. Dyn. 5, 283 (1977).

[29] M. Hwang and A. F. Arrieta, Energy harvesting characteristics in metamaterials based on bistable lattices, in Active and Passive Smart Structures and Integrated Systems XIII, edited by A. Erturk (SPIE, Bellingham, WA, 2019), p. 13.

[30] J. Thompson and H. Stewart, Nonlinear Dynamics and Chaos (Wiley, New York, 2002).

[31] T. Dauxois and M. Peyrard, Physics of Solitons (Cambridge University Press, Cambridge, England, 2006).

[32] H. Kulah and K. Najafi, Energy scavenging from lowfrequency vibrations by using frequency up-conversion for wireless sensor spplications, IEEE Sens. J. 8, 261 (2008).

[33] Both excitation types are very common in engineering applications. The force input is relevant for either rotating unbalanced mass or noncontacting sources, and the displacement input is relevant for a contacting excitation source, especially when its inertia is large such that the reaction force from the structure hardly affects the motion of the source.

[34] The next dominant branch appears at $\sim 24.5 \mathrm{~Hz}$, which is the second macroscopic structural mode.

[35] L. N. Virgin, Introduction to Experimental Nonlinear Dynamics (Cambridge University Press, Cambridge, England, 2000). 